\title{
Evaluation of enzyme biomarkers in freshwater invertebrates from Taro and Ticino river, Italy
}

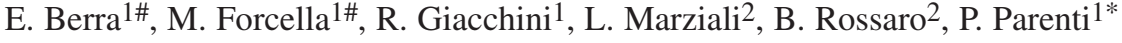 \\ ${ }^{1}$ Department of Environmental Sciences, University of Milano-Bicocca, Piazza della Scienza 1, 20126 Milano, Italy. \\ ${ }^{2}$ Department of Biology, University of Milano, via Celoria 26, 20133, Milano, Italy.
}

\begin{abstract}
Benthic macroinvertebrates are an essential component of freshwater environments. The ecotoxicological risk of benthic communities, estimated through the assay of biochemical markers, can be used as an early warning signal for environment alterations. In this work the activities of a number of enzymes, regarded as potential biomarker of exposure to pollutants (catalase, acetylcholinesterase, glutathione-S-transferase) was determined in homogenates of whole organisms. Specimens were collected in the rivers Taro and Ticino, northern Italy, in stony bottom reaches in five and three stations respectively. The orders of aquatic Insects Diptera, Plecoptera, Odonata, Ephemeroptera and Trichoptera, the Crustacea Amphipoda, and Annelida Oligochaeta were examined. Additional enzymes, such as NADP-dependent malate dehydrogenase, isocitric dehydrogenase, glucose-6phosphate dehydrogenase, alcohol dehydrogenase, and lactate dehydrogenase were considered in the study. Results emphasize significant differences among taxa concerning the specific activity of most enzymes.
\end{abstract}

Keywords : biomarkers, enzymes, invertebrates, insect larvae, chironomidae.

\section{Introduction}

Freshwater invertebrates are commonly used in biological monitoring, but research of biomarkers at biochemical level is still at the early stage of development. Biomarkers in aquatic species are regarded as important for detecting stressor components, such as presence of pollutants and changes in environmental factors (temperature, $\mathrm{pH}$, oxygen content, etc.). Hyne and Maher (2003) reviewed the application of biochemical measurements in invertebrates as a supplementary tool to predict changes in natural populations of aquatic invertebrates. They highlighted the lack of knowledge on the linkages between biochemical biomarker variations and macroinvertebrate population response.

Benthic invertebrates are one of the most important components of riverine ecosystems and a major food resource for fish. They are particularly attractive for

\footnotetext{
\# These authors contributed equally to this work. * Corresponding author : E-mail : paolo.parenti@unimib.it
}

biomarker measurement studies for several reasons, as: i) they are easily available, widely distributed, relatively abundant, and easy to collect; ii) many taxa are fairly sedentary and thus representative of local conditions; iii) they are closely associated with sediments; and iv) they are near the base of food chains (Hare 1992). In several programs emphasis has been given on analyzing changes in macroinvertebrate communities or population (e.g. Engel \& Voshell 2002, Ogbeibu \& Oribhabor 2002, Neumann et al. 2003, Sandin 2003). By contrast, studies using biochemical biomarkers have been carried out in organisms exposed to selected concentrations of chemicals under laboratory controlled conditions (Ibrahim et al. 1998, Kheir et al. 2001, De Coen et al. 2001, Hyne \& Maher 2003). These studies are helpful in establishing cause-effect relationships of specific contaminants and have been used to predict the effect of such contaminants on natural populations (Clements 2000).

Despite an extensive use of biochemical biomarkers in ecological risk assessments of aquatic ecosystems, studies on invertebrates in which the normal range of biomarker variability is determined are rare and, when 
present, they are focused on a single species (Olsen et al. 2001). The purpose of this paper is to present a general account of a series of enzyme activities currently recognized as biochemical biomarkers in macroinvertebrates from two Italian rivers, to give a basis for comparative biochemistry among examined taxa by including the determination of enzymes not currently included among biomarkers and evaluate the possible role of all the tested enzymes as useful biomarkers during seasonal changes of environmental conditions.

\section{Material and methods}

\section{Study area}

The Taro has a course of $125 \mathrm{~km}$ and flows from the northern Apennines (Penna mountain) into the Po. The river flows in a limestone bed, through a landscape changing from canopy cover upstream to meadows downstream. The discharge is up to $37 \mathrm{~m}^{3} \mathrm{~s}^{-1}$ during floods and it goes down to $1.5 \mathrm{~m}^{3} \mathrm{~s}^{-1}$ during low waters. The discharge was $2.2 \mathrm{~m}^{3} \mathrm{~s}^{-1}$ in July 2001 and 1.3 $\mathrm{m}^{3} \mathrm{~s}^{-1}$ in August 2001 at station 5. In 2002, river discharge was higher than in 2001, and in 2003 an exceptionally low water flow was observed. At the upstream station (Station 1, 44 ${ }^{\circ} 28^{\prime}$ Lat $9^{\circ} 33^{\prime}$ Long) the river flows in a narrow valley, in forested region (Quercus robur) and has a stony bottom with large rocks. At station 2 (4430' Lat 9 ${ }^{\circ} 39^{\prime}$ Long), the stony bottom consists of boulders and cobbles, with lower water velocity: here the Taro flows in an open region and receives seepage from the town of Compiano. At station 3, about $30 \mathrm{~km}$ downstream (44 $34^{\prime}$ Lat $9^{\circ} 55^{\prime}$ Long), it flows in a deep valley with a larger stony bottom river bed consisting of boulders, cobbles and pebbles. Station 4 ( $44^{\circ} 41^{\prime}$ Lat $10^{\circ} 4^{\prime}$ Long) is characterized by a large valley with a mixed gravel and sandy bottom; station 5 (44 $48^{\prime}$ Lat $10^{\circ} 11^{\prime}$ Long) has a sandy bottom with artificial rocks on the riverbank for flood protection.

The Ticino is a West tributary of the Po river and immediately after its outlet from the Maggiore lake it marks the border between Piedmont and Lombardy. The Ticino river has a length of $248 \mathrm{~km}$ and a drainage basin of $6599 \mathrm{~km}^{2}$; at its outlet from the Maggiore Lake it is forced to flow in a constrained segment, downstream it opens in a floodplain and flows as a braided river which divides into a main and secondary channels (3- 4 or more). A wide contact is established between water and riparian shore, the formation of fluvial isles is observed. The presence of the Maggiore Lake upstream guarantees a minimum flow, dumpens peak flows and supplies a high biological load. These combination of factors that are not readily found in other areas has favoured the presence of fish species not easily found in other areas, favouring the presence of fish species of high faunistic and economic value such as the grayling (Thymallus thymallus), encouraging to an attempt to reintroduce the otter. Interest in the high conservation value of the area has determined the creation in the 1978 of the Ticino Park Agency.

\section{Data collection}

The Taro river was investigated from August 2002 to December 2003, the Ticino river from January 2003 to December 2003. Measurements of water temperature, dissolved oxygen, $\mathrm{pH}$, conductivity were recorded using a field multiprobe in 5 stations in the Taro and in 3 stations in the Ticino river from the source to the confluence with the Po river. Water samples were also collected and analyzed for the main chemical parameters according to Standard Methods (APHA, 1992) and IRSA-CNR (1994): sulphate, nitrate nitrogen, ammonium nitrogen, orthophosphate and total phosphorous. Invertebrates were collected with a Surber net, sampling for about 15 minutes in stony bottom substrate. Samples were preserved in alcohol for the identification of taxa, the level of identification was family or genus, according to the different groups.

\section{Enzyme assay}

For each set of analysis, 0.5-3 $\mathrm{g}$ of larvae of similar size were gathered, transported alive into laboratory in a portable freezer which maintained about $5^{\circ} \mathrm{C}$, suspended and homogenized with 9 volumes of a hypotonic Hepes-Tris $10 \mathrm{mM}, \mathrm{pH} 7.5$, containing $1 \mathrm{mM}$ dithiothreitol. The suspension was then filtered through two layers of surgical gauze. Crude homogenate was centrifuged at $15,000 \mathrm{~g}$ at $4^{\circ} \mathrm{C}$ for $30 \mathrm{~min}$. The resulting supernatant was used to measure cytosolic activities of detoxification enzymes. For acetylcholinesterase (AChE) assay larvae were homogenized in the same buffer, except $1 \%$ Triton X-100, which offers the advantage of a high level of solubilization without denaturation. Enzymes were assayed using the following procedures. Isocitric dehydrogenase (ICDH), malic enzyme (EM), alcohol dehydrogenase (ADH), lactate dehydrogenase (LDH), and glucose-6-phosphate dehydrogenase (G6PDH) were assayed according to Bergmeyer et al. (1974). Catalase (CAT) was assayed according to Bergmeyer and Grassl (1983) using 12 $\mathrm{mM} \mathrm{H} \mathrm{O}_{2}$ as substrate and glutathione-S-transferase (GST) according to Habig et al. (1974). AChE was assayed according to Ikezawa and Taguchi (1981) in the presence of $0.5 \mathrm{mM}$ acetylthiocholine iodide. All as- 
says were performed in triplicate at $30^{\circ} \mathrm{C}$ in Cary 3 Spectrophotometer, recorded on personal computer, and analysed by the Cary Win UV application software for Windows 2000.

Activity was expressed in international units and referred to protein concentration as determined by the method of Bradford (1976).

\section{Results}

Tables 1 and 2 show the overall composition and distribution of benthic macroinvertebrates at the collection sites in Taro and Ticino rivers, respectively. In the Taro river the Chironomidae are the most abundant taxon, followed by Tabanidae and Tipulidae. Among Plecoptera, Perlidae, Perlodidae and Leuctridae, were in comparable abundance. Hydropsychidae and Ephemerellidae were the most abundant families of Trichoptera and Ephemeroptera respectively. In the Ticino river, Gammaridae and Lumbricidae were the most abundant taxa. Odonata Coenagrionidae were found in both rivers. The number of specimens belonging to each taxon varied greatly in space and time so that a lower number of taxa than that listed in Tables 1 and 2 allowed to have enough biomass to perform enzymatic analysis. The sampled stations were not significantly different in water temperature, oxygen content, $\mathrm{pH}$ and conductivity (Figs. 1 and 2). Heavy metal analysis performed on water samples collected at stations with higher biodiversity, namely stn. 1,2, and 3 of Taro rivers, revealed absence of lead and cadmium, whereas copper ranged from $6 \times 10^{-4}$ to $8 \times 10^{-4}$ p.p.m., chromium form $1 \times 10^{-4}$ to $6 \times 10^{-4}$ p.p.m., and zinc from $1 \times 10^{-4}$ to $6 \times 10^{-4}$ p.p.m. Heavy metals were then determined in Hydropsychidae larvae, taken as a reference for their presence in all sites. Results showed absence of chromium, whereas zinc concentration ranged from 1.15 to 2.83 p.p.m and copper concentration from 0.38 to 0.71 p.p.m., from station 1 to 3 , respectively.

The study of enzyme activities revealed striking dif-

Table 1. Distribution of benthic macroinvertebrates in Taro river sites. Animals were collected during the period January 2002 - December 2003. «+» = more than or equal to 3 individuals/month; « 3 individuals/month; «-»= absence.

\begin{tabular}{|c|c|c|c|c|c|}
\hline Taxon & Site 1 & Site 2 & Site 3 & Site 4 & Site 5 \\
\hline \multicolumn{6}{|l|}{ DIPTERA } \\
\hline Athericidae & + & \pm & - & - & - \\
\hline Chironomidae & + & + & + & + & + \\
\hline Limonidae & + & + & \pm & \pm & \pm \\
\hline Simulidae & + & \pm & \pm & \pm & + \\
\hline Tabanidae & + & + & & & \pm \\
\hline Tipulidae & + & + & \pm & \pm & \pm \\
\hline \multicolumn{6}{|l|}{ PLECOPTERA } \\
\hline Leuctridae & + & + & + & \pm & - \\
\hline Perlidae & + & + & \pm & - & - \\
\hline Perlodidae & + & + & + & \pm & - \\
\hline TRICHOPTERA & & & & & \\
\hline Hydropsychidae & + & + & + & + & + \\
\hline Limnephilidae & + & + & - & - & - \\
\hline Rhyacophilidae & + & + & \pm & \pm & - \\
\hline $\begin{array}{l}\text { Sericostomatidae } \\
\text { EPHEMEROPTERA }\end{array}$ & + & + & - & - & - \\
\hline Baetidae & + & + & + & + & + \\
\hline Ephemerellidae & + & + & + & + & + \\
\hline Heptageniidae & + & + & - & - & - \\
\hline $\begin{array}{l}\text { Leptophlebiidae } \\
\text { ODONATA }\end{array}$ & + & + & - & - & - \\
\hline Gomphidae & - & + & + & + & - \\
\hline
\end{tabular}


Table 2. Distribution of benthic macroinvertebrates in Ticino river sites. Animals were collected during the period January 2003 - December 2003. «+» = more than or equal to 3 individuals/month; $\ll \pm \ll=$ less than 3 individuals/month; «-» = absence.

\begin{tabular}{|c|c|c|c|}
\hline Taxon & Site 1 & Site 2 & Site 3 \\
\hline \multicolumn{4}{|l|}{ DIPTERA } \\
\hline Athericidae & + & \pm & - \\
\hline Chironomidae & + & + & + \\
\hline Limonidae & + & + & \pm \\
\hline Simulidae & + & \pm & \pm \\
\hline Tabanidae & + & + & \\
\hline Tipulidae & + & + & \pm \\
\hline \multicolumn{4}{|l|}{ PLECOPTERA } \\
\hline Leuctridae & + & + & + \\
\hline Perlidae & + & + & \pm \\
\hline Perlodidae & + & + & + \\
\hline \multicolumn{4}{|l|}{ TRICHOPTERA } \\
\hline Hydropsychidae & + & + & + \\
\hline Limnephilidae & + & + & - \\
\hline Rhyacophilidae & + & + & \pm \\
\hline Sericostomatidae & + & + & - \\
\hline \multicolumn{4}{|l|}{ EPHEMEROPTERA } \\
\hline Baetidae & + & + & + \\
\hline Ephemerellidae & + & + & + \\
\hline Heptageniidae & + & + & - \\
\hline Leptophlebiidae & + & + & - \\
\hline \multicolumn{4}{|l|}{ ODONATA } \\
\hline Gomphidae & - & + & + \\
\hline Coenagrionidae & - & + & + \\
\hline CRUSTACEA & & & \\
\hline Gammaridae & + & + & + \\
\hline ANNELIDA & & & \\
\hline Lumbricidae & + & + & + \\
\hline
\end{tabular}

ference among taxa and a lesser degree of temporal (seasonal) and spatial (among stations) variability within each taxon. In spite of some significant difference detected for individual enzymes within a taxon, the response was cumulated in a single value \pm S.E. and then reported in Tables 3 and 4. To avoid misinterpretation, larvae of the same size were used for each taxon for determination of enzyme activities.

Table 3 summarizes the data cumulated over the collection period in the five stations of the Taro river. Results show that, among the enzymes tested, catalase has the higher specific activity, with high variation from taxon to taxon. Values ranged from about 97 $\mathrm{U} / \mathrm{mg}$ in Leuctridae to about $4 \mathrm{U} / \mathrm{mg}$ in Odonata. Differences were observed among families of the same order (e.g. Chironomidae, Tabanidae and Tipulidae). Catalase value in a taxon ranged from a roughly constant value with variation usually below $50 \%$ (Perlidae, Leuctridae, Heptageniidae, and Chironomidae) to a quite fluctuant value well over $100 \%$ (Tipulidae, Hydropsychidae, Ephemerellidae, and Gomphidae). Major rise in activity was observed for most taxa in $\mathrm{Au}-$ tumn 2002 (e.g. Chironomidae, Tipulidae, Hydropsychidae, Ephemerellidae, Gomphidae). This pattern in the data were partially confirmed one year later (Autumn 2003) in which the catalase activity in several taxa increased compared to Summer 2003 (e.g. Tabanidae, Leuctridae, Heptageniidae). For most taxa the activity was also higher in Spring 2003 than in Winter 2002.

Acetylcholinesterase activity was highly variable in some taxa (Tipulidae, Hydropsychidae, Gomphidae, Heptageniidae) with temporal variability of 4-5 times. In other taxa AChE activity changed seasonally less 

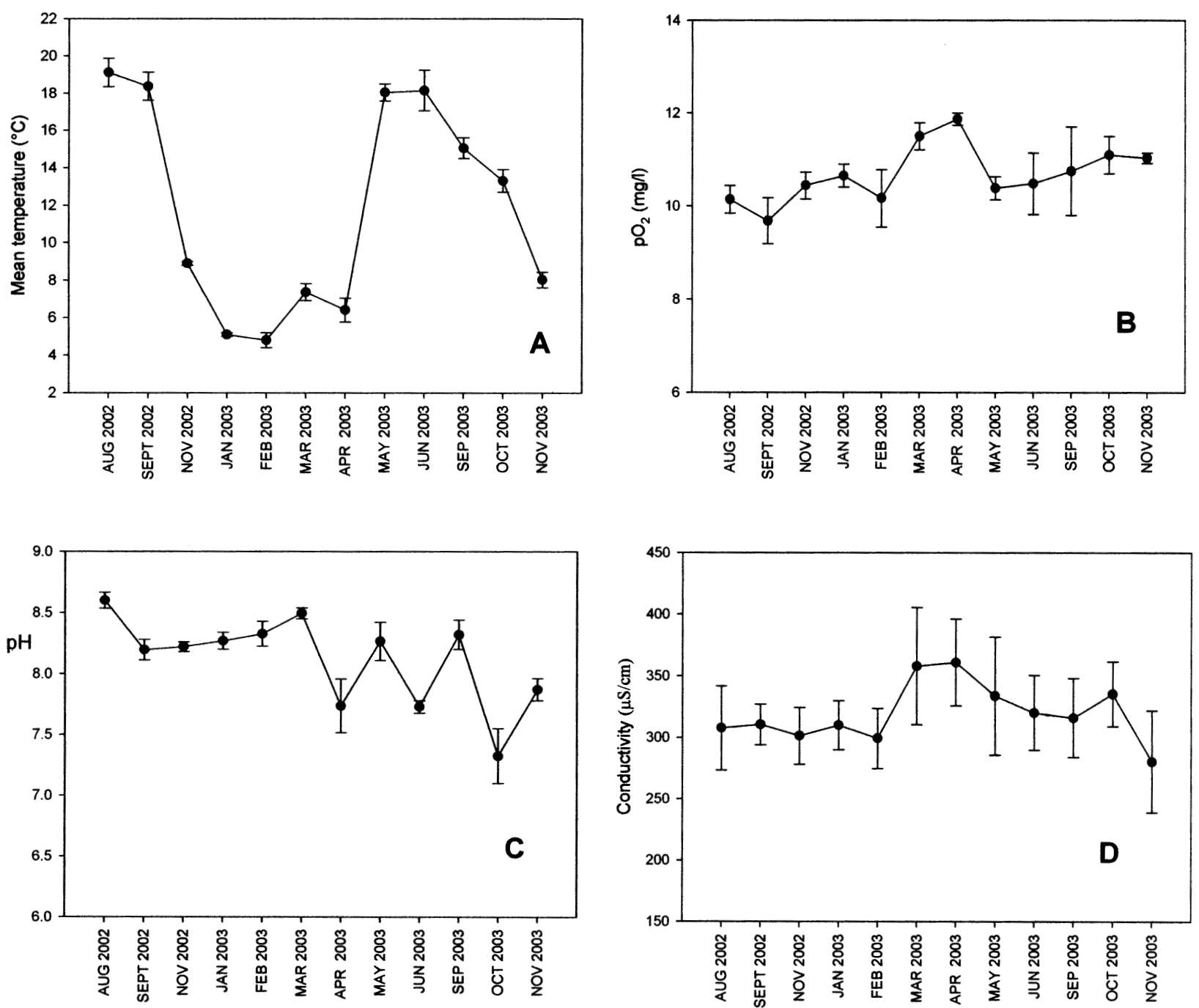

Fig. 1. Environmental parameters detected in five sampling stations along Taro river, Italy from August 2002 to November 2003. Mean temperature (A), oxygen dissolved (B), $\mathrm{pH}(\mathrm{C})$, and conductibility (D) are plotted.

than three times (Chironomidae, Tabanidae, Leuctridae), whereas Perlidae showed a fairly constant activity throughout the year. Raise and fall in AChE activity was not correlated with stations among taxa.

Taxa differed greatly in the activity of glutathione-Stransferase (GST). Gomphidae had activities in most instances one order of magnitude higher than other taxa or even larger when compared, for example, to Tabanidae. Values were temporally less variable within the same taxon than the two previous enzymes.
In order to determine any relationship between metabolically related enzyme activities, the data were cumulated, plotted, and analysed by regression analysis. A significant correlation was found in most taxa for catalase vs GST (Ephemeroptera, $p<0.0062$; Diptera, $p<0.0018$; Odonata, $p<0.0016$; Plecoptera, $p<0.0076$; Gammaridae, $p<0.0104)$, in only two taxa for AChE vs GST (Ephemeroptera, $p<0.0047$; Diptera, $p<0.0073$ ) and in all but Ephemeroptera for ME (or ICDH) vs GST (Fig. 2). For Diptera high levels of GST matched high specific activity of catalase, malic enzyme, 

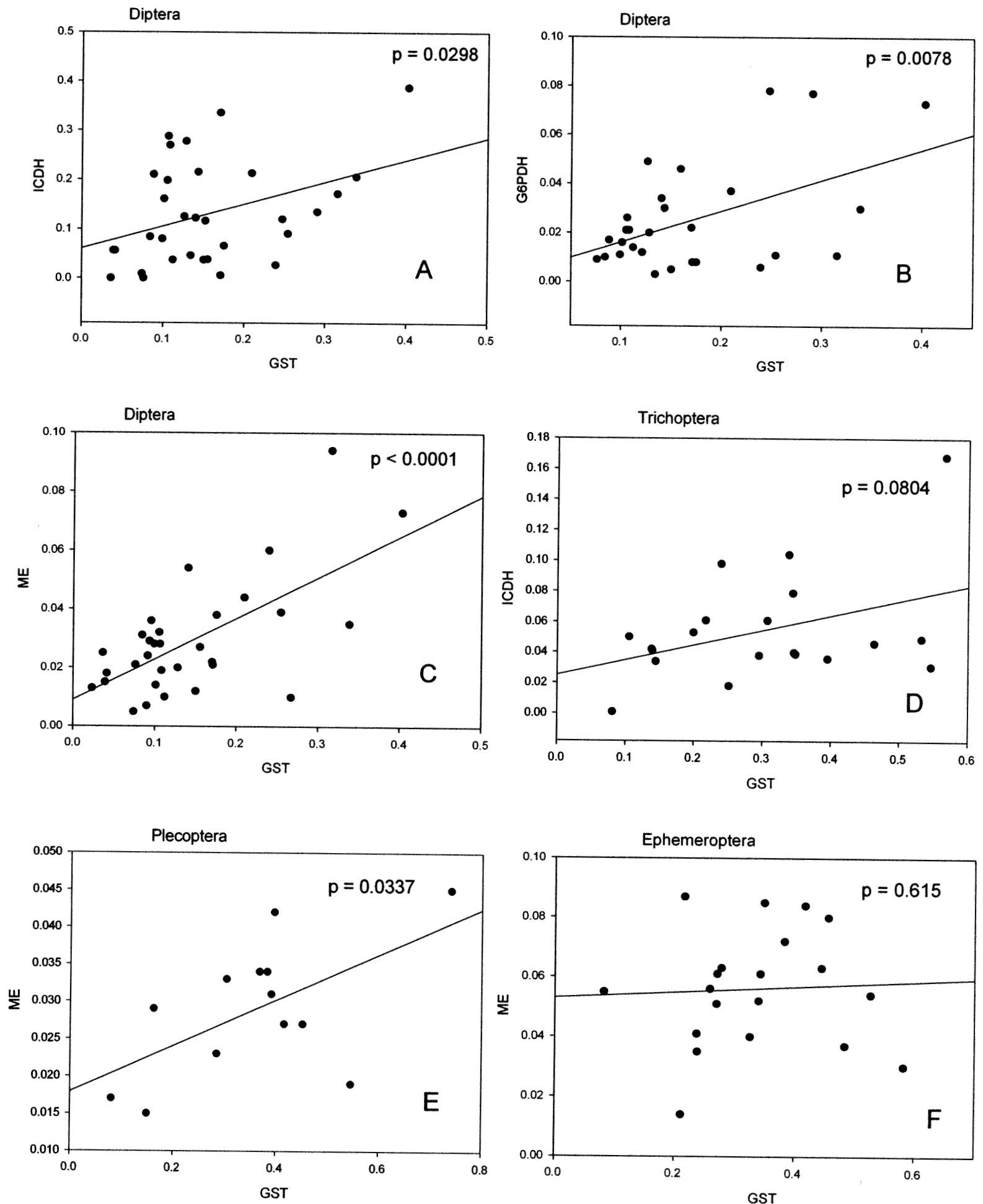

Fig. 2. Correlation between GST and NADPH-generating enzymes in insect larvae collected from Taro and Ticino river. The enzymes ICDH, G6PDH and ME in Diptera were plotted in panels A, B, and C, respectively. Enzymes in Trichoptera, Ephemeroptera, and Plecoptera were in panel D, E, and F respectively. Data were taken from Tables 3 and 4 . The $p$ value of regression analysis is indicated. 
Table 3. Enzyme activities in macroinvertebrates collected from Taro river, Italy. Numbers represent International Units of activity ( $\mu \mathrm{mol} / \mathrm{min} / \mathrm{mg}$ protein) and are means \pm S.E. of 3 to 6 independent samples each performed in triplicate; n.d., not detected; -, not determined.

\begin{tabular}{|c|c|c|c|c|c|c|c|}
\hline Enzyme & Taxon & $\begin{array}{c}\text { Summer } 2002 \\
\text { (Aug - Sept) }\end{array}$ & $\begin{array}{l}\text { Autumn } 2002 \\
\text { (Oct - Dic) }\end{array}$ & $\begin{array}{l}\text { Winter } 2003 \\
\text { (Jan - Mar) }\end{array}$ & $\begin{array}{l}\text { Spring } 2003 \\
\text { (Apr - Jun) }\end{array}$ & $\begin{array}{l}\text { Summer } 2003 \\
\text { (Sept) }\end{array}$ & $\begin{array}{c}\text { Autumn } 2003 \\
\text { (Oct - Nov) }\end{array}$ \\
\hline \multirow[t]{9}{*}{ CAT } & Chironomidae & $13.57 \pm 1.27$ & $20.26 \pm 0.98$ & $9.39 \pm 0.17$ & $12.06 \pm 0.820$ & - & - \\
\hline & Tabanidae & $8.24 \pm 0.59$ & $9.15 \pm 3.32$ & $6.79 \pm 0.01$ & $18.52 \pm 0.03$ & $11.39 \pm 1.29$ & $18.08 \pm 1.27$ \\
\hline & Tipulidae & - & $45.21 \pm 4.36$ & $40.28 \pm 3.17$ & $17.36 \pm 1.43$ & - & - \\
\hline & Perlidae & $31.57 \pm 0.47$ & $27.65 \pm 9.07$ & $27.72 \pm 0.58$ & $32.82 \pm 0.89$ & $29.96 \pm 0.93$ & $25.48 \pm 0.86$ \\
\hline & Leuctridae & $96.79 \pm 3.31$ & - & $54.59 \pm 3.56$ & - & $61.81 \pm 5.48$ & $88.32 \pm 1.24$ \\
\hline & Hydropsychidae & $13.15 \pm 0.51$ & $15.86 \pm 1.93$ & $8.47 \pm 0.26$ & $28.00 \pm 0.19$ & $18.40 \pm 1.14$ & $18.81 \pm 0.36$ \\
\hline & Ephemerellidae & - & $46.85 \pm 17.88$ & $27.26 \pm 0.81$ & $16.94 \pm 0.04$ & - & - \\
\hline & Heptageniidae & - & - & $19.39 \pm 0.17$ & $23.19 \pm 0.31$ & $21.89 \pm 1.6$ & $31.96 \pm 2.17$ \\
\hline & Gomphidae & $9.07 \pm 3.01$ & $22.85 \pm 1.57$ & - & $4.06 \pm 0.35$ & - & $5.42 \pm 0.30$ \\
\hline \multirow[t]{9}{*}{ AChE } & Chironomidae & - & $0.052 \pm 0.005$ & $0.019 \pm 0.001$ & $0.023 \pm 0.001$ & - & - \\
\hline & Tabanidae & $0.193 \pm 0.003$ & $0.271 \pm 0.036$ & - & $0.212 \pm 0.003$ & - & $0.501 \pm 0.009$ \\
\hline & Tipulidae & - & - & $0.122 \pm 0.001$ & - & - & $0.024 \pm 0.001$ \\
\hline & Perlidae & $0.105 \pm 0.005$ & $0.091 \pm 0.009$ & $0.109 \pm 0.003$ & $0.087 \pm 0.004$ & $0.101 \pm 0.006$ & $0.070 \pm 0.003$ \\
\hline & Leuctridae & $0.058 \pm 0.002$ & - & $0.091 \pm 0.003$ & - & $0.142 \pm 0.003$ & $0.102 \pm 0.029$ \\
\hline & Hydropsychidae & $0.243 \pm 0.007$ & $0.237 \pm 0.005$ & $0.069 \pm 0.002$ & - & $0.102 \pm 0.001$ & $0.054 \pm 0.001$ \\
\hline & Ephemerellidae & - & - & - & $0.140 \pm 0.003$ & - & - \\
\hline & Heptageniidae & - & - & $0.084 \pm 0.003$ & $0.119 \pm 0.001$ & $0.038 \pm 0.005$ & $0.166 \pm 0.002$ \\
\hline & Gomphidae & $0.067 \pm 0.001$ & $0.306 \pm 0.032$ & - & - & $0.073 \pm 0.002$ & $0.076 \pm 0.002$ \\
\hline \multirow[t]{9}{*}{ GST } & Chironomidae & $0.152 \pm 0.005$ & $0.128 \pm 0.021$ & $0.105 \pm 0.011$ & $0.091 \pm 0.001$ & - & - \\
\hline & Tabanidae & $0.039 \pm 0.002$ & $0.041 \pm 0.002$ & $0.052 \pm 0.003$ & $0.067 \pm 0.001$ & $0.014 \pm 0.001$ & $0.045 \pm 0.001$ \\
\hline & Tipulidae & - & $0.254 \pm 0.040$ & $0.215 \pm 0.019$ & $0.239 \pm 0.005$ & - & - \\
\hline & Perlidae & - & $0.416 \pm 0.037$ & $0.162 \pm 0.002$ & $0.166 \pm 0.004$ & $0.180 \pm 0.009$ & $0.261 \pm 0.001$ \\
\hline & Leuctridae & $0.580 \pm 0.018$ & - & $0.468 \pm 0.022$ & - & $0.288 \pm 0.022$ & $0.500 \pm 0.049$ \\
\hline & Hydropsychidae & $0.466 \pm 0.027$ & $0.084 \pm 0.005$ & $0.330 \pm 0.009$ & $0.097 \pm 0.004$ & $0.181 \pm 0.012$ & $0.200 \pm 0.004$ \\
\hline & Ephemerellidae & - & $0.527 \pm 0.046$ & $0.399 \pm 0.020$ & $0.290 \pm 0.009$ & - & - \\
\hline & Heptageniidae & - & - & $0.308 \pm 0.009$ & $0.325 \pm 0.004$ & $0.205 \pm 0.026$ & $0.358 \pm 0.005$ \\
\hline & Gomphidae & $2.356 \pm 0.007$ & $2.076 \pm 0.052$ & - & $1.217 \pm 0.010$ & - & $1.110 \pm 0.010$ \\
\hline \multirow[t]{9}{*}{ G6PDH } & Chironomidae & - & $0.020 \pm 0.003$ & $0.021 \pm 0.001$ & - & - & - \\
\hline & Tabanidae & n.d. & n.d. & $0.005 \pm 0.004$ & $0.011 \pm 0.001$ & - & - \\
\hline & Tipulidae & - & $0.011 \pm 0.003$ & $0.010 \pm 0.002$ & $0.006 \pm 0.001$ & - & - \\
\hline & Perlidae & - & $0.010 \pm 0.005$ & $0.003 \pm 0.001$ & n.d. & - & - \\
\hline & Leuctridae & n.d. & - & $0.006 \pm 0.003$ & - & - & - \\
\hline & Hydropsychidae & $0.017 \pm 0.006$ & $0.002 \pm 0.001$ & $0.002 \pm 0.002$ & - & - & - \\
\hline & Ephemerellidae & - & n.d. & $0.003 \pm 0.001$ & - & - & - \\
\hline & Heptageniidae & - & - & n.d. & n.d. & - & - \\
\hline & Gomphidae & $0.004 \pm 0.004$ & $0.003 \pm 0.003$ & - & - & - & - \\
\hline \multirow[t]{9}{*}{$\mathbf{E M}$} & Chironomidae & - & $0.020 \pm 0.001$ & $0.036 \pm 0.002$ & $0.027 \pm 0.003$ & - & - \\
\hline & Tabanidae & $0.015 \pm 0.001$ & $0.018 \pm 0.001$ & $0.023 \pm 0.002$ & $0.027 \pm 0.005$ & - & - \\
\hline & Tipulidae & - & $0.039 \pm 0.001$ & $0.066 \pm 0.008$ & $0.060 \pm 0.001$ & - & - \\
\hline & Perlidae & - & $0.027 \pm 0.001$ & $0.025 \pm 0.005$ & $0.036 \pm 0.006$ & - & - \\
\hline & Leuctridae & n.d. & - & $0.036 \pm 0.009$ & - & - & - \\
\hline & Hydropsychidae & $0.053 \pm 0.001$ & $0.077 \pm 0.008$ & $0.045 \pm 0.008$ & $0.041 \pm 0.004$ & - & - \\
\hline & Ephemerellidae & - & $0.054 \pm 0.003$ & $0.078 \pm 0.006$ & $0.060 \pm 0.005$ & - & - \\
\hline & Heptageniidae & - & - & $0.059 \pm 0.008$ & $0.057 \pm 0.003$ & - & - \\
\hline & Gomphidae & n.d. & $0.002 \pm 0.002$ & - & n.d. & - & - \\
\hline \multirow[t]{9}{*}{ ICDH } & Chironomidae & $0.116 \pm 0.006$ & $0.278 \pm 0.014$ & $0.198 \pm 0.004$ & - & - & - \\
\hline & Tabanidae & $0.056 \pm 0.003$ & $0.056 \pm 0.001$ & 0 & $0.082 \pm 0.002$ & - & - \\
\hline & Tipulidae & - & $0.091 \pm 0.001$ & $0.119 \pm 0.005$ & $0.027 \pm 0.001$ & - & - \\
\hline & Perlidae & $0.009 \pm 0.001$ & $0.025 \pm 0.001$ & $0.006 \pm 0.004$ & $0.021 \pm 0.001$ & - & - \\
\hline & Leuctridae & n.d. & - & 0 & - & - & - \\
\hline & Hydropsychidae & $0.072 \pm 0.003$ & $0.024 \pm 0.003$ & $0.047 \pm 0.007$ & - & - & - \\
\hline & Ephemerellidae & - & n.d. & $0.005 \pm 0.005$ & - & - & - \\
\hline & Heptageniidae & - & - & n.d. & n.d. & - & - \\
\hline & Gomphidae & $0.019 \pm 0.005$ & $0.009 \pm 0.001$ & - & - & - & - \\
\hline
\end{tabular}


Table 4. Enzyme activities in macroinvertebrates collected from Ticino river, Italy. Numbers represent International Units of activity $(\mu \mathrm{mol} / \mathrm{min} / \mathrm{mg}$ protein $)$ and are means \pm S.E. of 3 to 7 independent samples each performed in triplicate; $\langle$ n.d.» $=$ not detected; $\ll-»=$ not determined.

\begin{tabular}{|c|c|c|c|c|c|}
\hline Enzyme & Taxon & $\begin{array}{l}\text { Winter } 2003 \\
\text { (Jan - Mar) }\end{array}$ & $\begin{array}{c}\text { Spring } 2003 \\
\text { (Apr - Jun) }\end{array}$ & $\begin{array}{l}\text { Summer } 2003 \\
\text { (Jul- Sept) }\end{array}$ & $\begin{array}{c}\text { Autumn } 2003 \\
\text { (Oct - Nov) }\end{array}$ \\
\hline \multirow[t]{12}{*}{ CAT } & Chironomidae & $11.51 \pm 0.28$ & $20.82 \pm 1.89$ & - & - \\
\hline & Tabanidae & $19.01 \pm 0.15$ & $16.06 \pm 0.69$ & $16.01 \pm 0.47$ & $19.43 \pm 0.02$ \\
\hline & Tipulidae & $14.19 \pm 0.66$ & $23.07 \pm 0.58$ & $58.98 \pm 0.78$ & $24.78 \pm 0.21$ \\
\hline & Hydropsychidae & $28.50 \pm 0.48$ & $31.51 \pm 0.38$ & $34.19 \pm 0.01$ & $24.97 \pm 0.59$ \\
\hline & Rhyacophilidae & $22.00 \pm 0.35$ & $29.01 \pm 0.25$ & $33.18 \pm 0.72$ & - \\
\hline & Ephemerellidae & $16.30 \pm 1.06$ & $14.19 \pm 0.66$ & $8.9 \pm 0.92$ & - \\
\hline & Heptageniidae & $26.45 \pm 1.80$ & $36.22 \pm 1.07$ & - & $20.88 \pm 1.5$ \\
\hline & Baetidae & $25.78 \pm 3.30$ & $35.78 \pm 1.30$ & - & - \\
\hline & Gomphidae & $4.36 \pm 0.50$ & $2.36 \pm 0.01$ & $8.37 \pm 0.44$ & - \\
\hline & Coenagrionidae & - & - & $35.88 \pm 4.94$ & - \\
\hline & Gammaridae & - & $6.60 \pm 0.15$ & $4.67 \pm 0.12$ & $4.05 \pm 0.21$ \\
\hline & Lumbricidae & - & $10.74 \pm 0.04$ & $7.46 \pm 0.10$ & $11.1 \pm 0.63$ \\
\hline \multirow[t]{12}{*}{ AChE } & Chironomidae & $0.014 \pm 0.000$ & $0.042 \pm 0.001$ & - & - \\
\hline & Tabanidae & $0.149 \pm 0.016$ & $0.085 \pm 0.005$ & $0.215 \pm 0.080$ & - \\
\hline & Tipulidae & $0.078 \pm 0.003$ & $0.066 \pm 0.003$ & $0.093 \pm 0.004$ & $0.082 \pm 0.003$ \\
\hline & Hydropsychidae & $0.028 \pm 0.001$ & $0.087 \pm 0.004$ & $0.114 \pm 0.002$ & $0.086 \pm 0.001$ \\
\hline & Rhyacophilidae & $0.022 \pm 0.001$ & $0.027 \pm 0.000$ & $0.033 \pm 0.000$ & - \\
\hline & Ephemerellidae & - & $0.070 \pm 0.002$ & $0.127 \pm 0.008$ & - \\
\hline & Heptageniidae & $0.061 \pm 0.001$ & $0.074 \pm 0.002$ & - & $0.148 \pm 0.000$ \\
\hline & Baetidae & $0.140 \pm 0.003$ & $0.132 \pm 0.008$ & - & - \\
\hline & Gomphidae & $0.089 \pm 0.015$ & $0.092 \pm 0.011$ & $0.037 \pm 0.002$ & $0.078 \pm 0.003$ \\
\hline & Coenagrionidae & - & - & $0.120 \pm 0.008$ & $0.116 \pm 0.005$ \\
\hline & Gammaridae & $0.129 \pm 0.003$ & $0.146 \pm 0.002$ & $0.307 \pm 0.005$ & $0.142 \pm 0.001$ \\
\hline & Lumbricidae & $0.384 \pm 0.020$ & $0.367 \pm 0.003$ & - & $0.280 \pm 0.012$ \\
\hline \multirow[t]{12}{*}{ GST } & Chironomidae & $0.245 \pm 0.001$ & $0.147 \pm 0.010$ & - & - \\
\hline & Tabanidae & $0.119 \pm 0.010$ & $0.118 \pm 0.004$ & $0.033 \pm 0.003$ & $0.161 \pm 0.001$ \\
\hline & Tipulidae & $0.149 \pm 0.020$ & $0.155 \pm 0.005$ & $0.042 \pm 0.003$ & $0.276 \pm 0.004$ \\
\hline & Hydropsychidae & $0.227 \pm 0.003$ & $0.140 \pm 0.006$ & $0.111 \pm 0.005$ & $0.222 \pm 0.012$ \\
\hline & Rhyacophilidae & $0.211 \pm 0.011$ & $0.140 \pm 0.001$ & $0.296 \pm 0.016$ & - \\
\hline & Ephemerellidae & $0.329 \pm 0.050$ & $0.313 \pm 0.015$ & $0.334 \pm 0.059$ & - \\
\hline & Heptageniidae & $0.304 \pm 0.002$ & $0.240 \pm 0.007$ & - & $0.354 \pm 0.016$ \\
\hline & Baetidae & $0.385 \pm 0.028$ & $0.449 \pm 0.033$ & - & - \\
\hline & Gomphidae & $1.129 \pm 0.003$ & $0.863 \pm 0.050$ & $1.415 \pm 0.122$ & - \\
\hline & Coenagrionidae & $4.359 \pm 0.084$ & - & $0.965 \pm 0.114$ & - \\
\hline & Gammaridae & $0.711 \pm 0.036$ & $0.454 \pm 0.003$ & $0.281 \pm 0.026$ & $0.251 \pm 0.010$ \\
\hline & Lumbricidae & $0.171 \pm 0.010$ & $0.086 \pm 0.004$ & $0.056 \pm 0.003$ & $0.174 \pm 0.001$ \\
\hline
\end{tabular}


Table 4. (Continued)

\begin{tabular}{|c|c|c|c|c|c|}
\hline Enzyme & Taxon & $\begin{array}{c}\text { Winter } 2003 \\
\text { (Jan - Mar) }\end{array}$ & $\begin{array}{l}\text { Spring } 2003 \\
\text { (Apr - Jun) }\end{array}$ & $\begin{array}{l}\text { Summer } 2003 \\
\quad \text { (Jul- Sept) }\end{array}$ & $\begin{array}{c}\text { Autumn } 2003 \\
\text { (Oct - Nov) }\end{array}$ \\
\hline \multirow[t]{12}{*}{ G6PDH } & Chironomidae & $0.068 \pm 0.01$ & $0.035 \pm 0.001$ & - & - \\
\hline & Tabanidae & n.d. & n.d. & - & - \\
\hline & Tipulidae & n.d. & $0.002 \pm 0.000$ & - & - \\
\hline & Hydropsychidae & $0.015 \pm 0.009$ & - & - & - \\
\hline & Rhyacophilidae & $0.029 \pm 0.006$ & - & - & - \\
\hline & Ephemerellidae & n.d. & n.d. & - & - \\
\hline & Heptageniidae & n.d. & n.d. & - & - \\
\hline & Baetidae & $0.003 \pm 0.001$ & n.d. & - & - \\
\hline & Gomphidae & n.d. & n.d. & - & - \\
\hline & Coenagrionidae & $0.007 \pm 0.001$ & n.d. & - & - \\
\hline & Gammaridae & n.d. & n.d. & - & - \\
\hline & Lumbricidae & $0.009 \pm 0.000$ & $0.010 \pm 0.004$ & - & - \\
\hline \multirow[t]{12}{*}{ EM } & Chironomidae & - & $0.054 \pm 0.003$ & - & - \\
\hline & Tabanidae & - & $0.005 \pm 0.001$ & - & - \\
\hline & Tipulidae & - & $0.027 \pm 0.001$ & - & - \\
\hline & Hydropsychidae & - & $0.042 \pm 0.001$ & - & - \\
\hline & Rhyacophilidae & - & $0.084 \pm 0.006$ & - & - \\
\hline & Ephemerellidae & - & $0.060 \pm 0.004$ & - & - \\
\hline & Heptageniidae & - & $0.087 \pm 0.011$ & - & - \\
\hline & Baetidae & - & $0.070 \pm 0.008$ & - & - \\
\hline & Gomphidae & - & $0.003 \pm 0.001$ & - & - \\
\hline & Coenagrionidae & - & $0.005 \pm 0.001$ & - & - \\
\hline & Gammaridae & - & $0.008 \pm 0.004$ & - & - \\
\hline & Lumbricidae & - & $0.018 \pm 0.003$ & - & - \\
\hline \multirow[t]{12}{*}{ ICDH } & Chironomidae & $0.127 \pm 0.004$ & $0.122 \pm 0.005$ & - & - \\
\hline & Tabanidae & - & $0.009 \pm 0.001$ & - & - \\
\hline & Tipulidae & - & $0.038 \pm 0.003$ & - & - \\
\hline & Hydropsychidae & $0.058 \pm 0.020$ & $0.034 \pm 0.001$ & - & - \\
\hline & Rhyacophilidae & $0.057 \pm 0.004$ & $0.061 \pm 0.019$ & - & - \\
\hline & Ephemerellidae & n.d. & n.d. & - & - \\
\hline & Heptageniidae & n.d. & n.d. & - & - \\
\hline & Baetidae & n.d. & n.d. & - & - \\
\hline & Gomphidae & - & $0.030 \pm 0.002$ & - & - \\
\hline & Coenagrionidae & n.d. & - & - & - \\
\hline & Gammaridae & n.d. & n.d. & - & - \\
\hline & Lumbricidae & $0.126 \pm 0.014$ & $0.011 \pm 0.001$ & - & - \\
\hline
\end{tabular}


ICDH, and G6PDH. Similar data were obtained for Plecoptera, except that ICDH and G6PDH were not included due to their very low or undetectable activity. In Ephemeroptera, which were characterized by the highest level of malic enzyme among the taxa examined, a positive correlation was found only between GST vs catalase or GST vs AChE, as in Odonata. A positive correlation of GST vs AChE was found only in Ephemeroptera, whereas in Diptera the correlation was negative. Absence of correlation was usually not shown in Figure 2, except that some plots were added for comparison (i.e, in Trichoptera a significant correlation was never found).

The activity of GST correlated with those NADPHgenerating enzymes (G6PDH, malic enzyme and ICDH) (Fig. 4), which, however, were not detected in all taxa. All but Gomphidae showed well-detectable activity of malic enzyme. Several taxa were found to have the ability to synthesize NADPH via isocitric dehydrogenase, an enzyme very active in Chironomidae but virtually absent in Leuctridae, Heptageniidae and Ephemerellidae. Glucose-6-phosphate dehydrogenase had very low or undetectable activity in most taxa, whereas Chironomidae had the highest specific activity for this enzyme (Tables 3 and 4).

\section{Discussion}

Biomarkers that employ enzyme activity measurements to detect sublethal levels of pollution are increasingly used in ecological risk assessments of aquatic ecosystems. These biomarkers have the capacity to identify the incidence of exposure to, and effect caused by xenobiotics such as pesticides, so providing an early warning of potentially damaging effects at higher levels of biological organization. Biochemical changes include the induction of detoxification enzymes that are capable of degrading the xenobiotic or the reduction in activity of enzymes sensitive to inhibition by the xenobiotic (Callaghan et al. 2002).

Aquatic organisms exposed in vivo, to conditions or xenobiotics, likely to enhance oxyradicals fluxes develop a metabolic response involving components of the cellular antioxidant system. These defenses are in general highly modulated: levels and activities of antioxidants are responsive to changes in the environmental milieu that affects the cellular production of activated oxygen species (Di Giulio et al. 1995).

Hydrogen peroxide is a normal product of oxygen metabolism in cells (Chance et al. 1979). If not eliminated, it can cause detrimental cytosolic effect, by reacting with superoxide $\left(\mathrm{O}_{2}-^{-}\right)$radicals in the presence of iron to produce hydroxyl radicals $\left(\mathrm{OH}^{\circ}\right)$.

Hydroxyl radicals can potentially cause a variety of deleterious changes in biological systems. The enzymatic degradation of hydrogen peroxide is catalysed by catalase and glutathione peroxidase (Gpox). The latter enzyme is, however, absent in insects (Mathews et al., 1997). In this work we demonstrated that catalase is highly active in macro invertebrates with specific activity ranging from about 4 to almost $100 \mathrm{U} / \mathrm{mg}$, ranging from Odonata to Plecoptera, respectively. As expected there is no differences in enzyme activity for the same taxon collected from two river system, but there are seasonal variations, which apparently are independent of environmental parameters. Interestingly, Plecoptera, which includes species requiring well-oxygenated water flows, was found to have the highest specific activity suggesting a protective role of this enzyme against oxidative stress.

The glutathione S-transferases (GSTs) are a family of biotransformation enzymes involved in conjugating a variety of lipophilic contaminant to - $\mathrm{SH}$ group of glutathione, neutralizing their electrophilic sites and rendering the products more water-soluble and less toxic, so allowing easier excretion from cells after further metabolism. Glutathione S-transferases have attracted attention in insects because of their involvement in the defence towards insecticides, particularly organophosphates, organochlorines and cyclodienes (Kostaropoulos et al. 2001). The reduction of the oxidized form of glutathione (GSSG) is brought about by the NADPH-specific GSSG reductase. NADPH is synthesized from the isocitrate dehydrogenase (ICDH), malic enzyme (ME) and glucose-6-phosphate dehydrogenase (G6PDH). The isocitrate dehydrogenase (ICDH) catalyses the oxidative decarboxylation of isocitrate to 2-oxoglutarate. Two ICDH types can be distinguished in vertebrates, insects and mollusks as follows. The NAD-specific ICDH from mitochondria and the NADP-specific ICDH, which in animals is present as different cytosolic and mitochondrial isoenzymes. The differing intracellular distribution of the two enzyme types led to the suggestion that NADP-ICDH is not really an enzyme of the citric acid cycle but delivers NADPH for biosynthetic purposes. Malic enzyme (ME) catalyses the oxidative decarboxylation of malate to pyruvate with the reduction of $\mathrm{NADP}^{+}$to NAD$\mathrm{PH}$. The enzyme may also uses $\mathrm{NAD}^{+}$as electron acceptor. Most cytoplasmatic and mitochondrial MEs prefer $\mathrm{NADP}^{+}$as coenzyme, but NAD-specific enzyme is always mitochondrial (Urich, 1990). Glucose-6- 
phosphate dehydrogenase (G6PDH) is a cytosolic enzyme and catalyses the conversion of glucose-6-phosphate to 6-phosphogluconate with the contemporary generation of NADPH from NADP ${ }^{+}$. As shown in Table 3 and 4 not all three enzymes can be detected at significant specific activity $(>0.001 \mathrm{U} / \mathrm{mg})$ in all taxa. In particular, Diptera differed from other taxa for bearing all three dehydrogenases, with Chironomidae having the highest activity for two of them (G6PDH and ICDH). Malic enzyme was quite evenly distributed among taxa, except in Odonata, whose biochemical features are surprisingly different from the other taxa. In fact, Odonata displayed the highest GST specific activity (1-2 order of magnitude higher that in other taxa), without a corresponding high level of NADPdependent dehydrogenases supposed to support the activity of GST. This intriguing result was shared also by Gammaridae (found only in Ticino river) and it was not dependent on assay conditions. We believe that intermediate metabolism in these organisms deserves further investigations. NADP-dependent dehydrogenase activity for most of the study taxa was well correlated with high GST activity, except for Trichoptera, in which a positive correlation was observed but was not significant, and Ephemeroptera, where the two parameters appeared not correlated.

Acetylcholinesterase (AChE) is a carboxylesterase enzyme important in the maintenance of normal nerve function, hydrolysing the neurotransmitter acetylcholine in cholinergic synapses. AChE is the primary target of neurotoxic pesticides such as organophosphates and carbamates designed to control invertebrate pests (Hassall, 1990). Inhibition resulting from irreversible binding at the AChE active site leads to the accumulation of acetylcholine in the synapse, resulting in the disruption of normal function. The measurement of AChE activity to detect inhibition resulting from pesticide binding has been used successfully in several laboratory studies (Crane et al. 1995, Ibrahim et al. 1998). It has been reported (Olsen et al. 2001) that the enzyme is subjected to a certain degree of variability, which could disturb the interpretation of field study. In the present study the specific activity of AChE changed on more than one occasion for several taxa. For example, in the majority of taxa from Ticino river the activity recorded in Spring 2003 was lower than that measured in organisms collected in Summer 2003. A further drop was then observed in Autumn 2003 and this pattern was partially observed in specimens collected in Taro river. These data might suggest that taxa responded at different degrees when they enter in contact with substances able to interfere with AChE activity. In particular, the sensitivity of the enzyme in various species could be different due to its different inhibition constant. This aspect requires laboratory studies including dose-response curves with appropriate AChE inhibitors. A series of experiments are presently in progress to answer this question.

In conclusion, the data reported in the present work represent the first attempt to monitoring basal levels of three widely recognized enzymatic biomarkers, catalase, GST and AChE, in benthic macroinvertebrates from two riverine communities. The utility of these biomarkers is far from being well defined, mainly because their use as biochemical markers has been largely neglected (Hyne \& Maher 2003). Our results show that: i) their specific activity differs markedly among different taxa of the same community, and ii) high specific activity of one of them is rarely accompanied by a corresponding variation of the level of an enzyme. Even though the responsiveness of some of these biomarkers is regarded to be low in freshwater invertebrates (Blat et al. 1988), this study showed that the responsiveness of the enzyme could be different from taxon to taxon from the two river systems. Therefore, to understand the importance of selected enzymes as useful biomarker in aquatic invertebrates, we need to increase our knowledge of their relationships with other enzymes activities not in only in a single taxon, but in a range of taxa as large as possible.

\section{Acknowledgements}

We are grateful to Dr. Maria Tringali for the determination of heavy metals and to the anonymous reviewers who helped to improve this article. This research was supported by a grant from MIUR entitled «An analysis of spatio-temporal distribution of species and populations belonging to critical groups living in inland waters, with morphological and molecular characterization 》n. 2002058154_002 PRIN 2002.

\section{References}

Bergmeyer H.U., Gawehn K. \& Grassl M. 1974. - Enzymes as biochemical reagents. Pages 427-522 in Bergmeyer H.U. (Ed.). $\mathrm{Me}$ thods of enzymatic analysis. Volume 1. Academic Press, New York and London.

Bergmeyer H.U. \& Grassl M. 1983. - Enzymes. Catalase. Pages 165166 in Bergmeyer H.U. (Ed.). Methods of enzymatic analysis. Volume 2. Academic Press, New York and London.

Blat A., Almar M.M. \& Romero F.J. 1988. - The effect of two sulfur containing pesticides, fenitrothion and endosulfan, on glutathione (GSH) content and on GSH S-transferase and $\gamma$-glutamyltranspeptidase activities in midgut gland of the American red crayfish Procambarus clarkii. Drug Metab. Drug Interact., 6, 383-394.

Bradford M.M. 1976. - A rapid and sensitive method for the quantization of protein utilizing the principle of dye-protein binding. Anal. Biochem. 72, 248-254.

Callaghan A., Fisher T.C., Grosso A., Holloway G. J. \& Crane M. 2002. - Effect of temperature and Pirimiphos methyl on biochemical biomarkers in Chironomus riparius Meigen. Ecotoxicol. Environ. Saf., 52, 128-133. 
Chance B., Seis H. \& Boveris A. 1979. - Hydroperoxide metabolism in mammalian organs. Physiol. Rev., 59, 527-603.

Clements W.H. 2000. - Integrating effects of contaminants across levels of biological organization: an overview. J. Aquat. Ecosyst. Stress Recovery, 7, 113-116.

Crane M., Delaney P., Watson S., Parker P. \& Walker C. 1995. - The effect of malathion 60 on Gammarus pulex (L.) below watercress beds. Environ. Toxicol. Chem., 14, 1181-1187.

De Coen W.M., Janssen C.R. \& Segner H. 2001. - The use of biomarkers in Daphnia magna toxicity testing V. In vivo alterationa in the carbohydrate metabolism of Daphnia magna exposed to sublethal conecntrations of Mercury and lindane. Ecotoxicol. Environm. Saf., 48, 230-234.

Di Giulio R.T., Benson W.H., Sanders B.M. \& Van Vield P.A. 1995 - Biochemical mechanisms: metabolism, adaptation, and toxicity. Pages 523-561 in Rand G.M. (Ed.). Fundamentals of aquatic toxicology: Effects, Environmental Fate, and Risk Assessment. Ecological Services Inc., North Palm Beach, Florida.

Engel S.R. \& Voshell J.R. Jr. 2002. - Volunteer Biological Monitoring: Can It Accurately Assess the Ecological Condition of Streams? Am. Entomol., 48, 164-177.

Habig W.H., Pabst M. J. \& Jakoby W.B. 1974. - Glutathione STransferases. The first enzymatic step in mercapturic acid formation. J. Biol. Chem., 249, 7130-7139

Hare L. 1992. - Aquatic insects and trace metals: Bioavailability, bioaccumulation and toxicology. Crit. Rev. Toxicol., 22, 327-369.

Hassal K. 1990. - The Biochemistry and Uses of Pesticides. Macmillan, London, $536 \mathrm{p}$.

Hyne R.V. \& Maher W.A. 2003. - Invertebrate biomarkers: links to toxicosis that predict population decline. Ecotoxicol. Environ. Saf., 54, 366-74

Ibrahim H., Kheir R., Helmi S., Lewis J. \& Crane M. 2001. - The effects of organophosphorus, carbamate, pyrethroid and organochlorine pesticides and a heavy metal on survival and cholinesterase activity of Chironomus riparius Meigen exposed to chemical-spiked sediments. Bull. Environ. Contam. Toxicol., 60, 448455.
Ikezawa H, Taguchi R. 1981. - Phosphatidylinositol-specific phospholipase $\mathrm{C}$ from Bacillus cereus and Bacillus thuringiensis. Pages 731-741 in Colowick N.P (Ed.). Methods Enzymology.

Kheir R., Ibrahim H., Lewis J., Callaghan A. \& Crane M. 2001. Comparison of acetylcholinesterase and glutathione S-transferase activity in Chironomus riparius Meigen exposed to chemical-spiked sediments. Bull. Environ. Contam. Toxicol., 66, 603-610.

Kostaropoulos I., Papadopoulos A.I., Metaxakis A., Boukouvala E. \& Papadopoulou-Mourkidou E. 2001. - Glutathione S-transferase in the defence against pyrethroids in insects. Insect Biochem. Mol. Biol., 31, 313-319.

Mathews M.C., Summers C.B. \& Felton G.W. 1997. - Ascorbate peroxidase: a novel antioxidant enzyme in insects. Arch. Insect Biochem. Physiol., 34, 57-68.

Neumann M., Liess M. \& Schulz R. 2003. - An expert system to estimate the pesticide contamination of small streams using benthic macroinvertebrates as bioindicators. Part 1. The database of LIMPACT. Ecol. Indicators, 2, 379-389.

Ogbeibu A.E. \& Oribhabor B.J. 2002. - Ecological impact of river impoundment using benthic macro-invertebrates as indicators. Water Res., 36, 2427-2436.

Olsen T., Ellerbeck L., Fisher T., Callaghan A. \& Crane M. 2001. Variability in acetylcholinesterase and glutathione S-transferase activities in Chironomus riparius Meigen deployed in situ at uncontaminated field sites. Environ. Toxicol. Chem., 20, 1725-1732.

Sandin L. 2003. - Benthic macroinvertebrates in Swedish streams: community structure, taxon richness, and environmental relations. Ecography, 26, 269-282.

Urich K. 1990. - Comparative Animal Biochemistry. Springer-Verlag, Berlin, $782 \mathrm{p}$. 